\title{
The COSEVAST Study Outcome: Evidence of COVID-19 Severity Proportionate to Surge in Arterial Stiffness
}

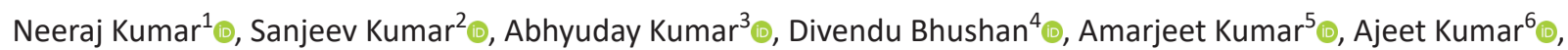 \\ Veena Singh ${ }^{7} \odot$, Prabhat K Singh ${ }^{8} \odot$
}

\begin{abstract}
Background: The severe acute respiratory syndrome coronavirus 2 (SARS-CoV-2) infection affects the cardiovascular system at many levels. It initially infects endothelial cells, inducing marked endothelial damage and inflammation. However, there was no empirical evidence of functional compromise of arterial walls.

Aims and objective: Our primary objective was to study functional arterial damage in coronavirus disease 2019 (COVID-19) and establish the noninvasive measurement of arterial stiffness as an independent marker of disease severity.

Materials and methods: We recorded the arterial stiffness of 23 mild, 21 moderate, and 20 severe COVID-19 patients grouped on the latest National Institute of Health (NIH) severity criteria. We observed arterial stiffness of COVID-19 patients with standard parameters like noninvasive estimated carotid-femoral pulse wave velocity (cfPWV), age-normalized increase in cfPWV (ANI_cfPWV), age-normalized increase in aortic augmentation pressure (ANI_AugP), and heart rate-normalized augmentation index (HRN_Alx). All the parameters were also corrected for statistically significant confounding factors.

Results: Moderate and severe COVID-19 patients have extremely significantly elevated arterial stiffness than mild patients. In mild patients, cfPWV $(829.1 \pm 139.2 \mathrm{~cm} / \mathrm{second})$ was significantly lower than both moderate $(1067 \pm 152.5 \mathrm{~cm} / \mathrm{second}, p<0.0001)$ and severe $(1416 \pm 253.9 \mathrm{~cm} / \mathrm{second}$, $p<0.0001)$ patients. ANI_cfPWV in moderate and severe patients was significantly higher than mild patients (mild: $101.2 \pm 126.1 \mathrm{~cm} / \mathrm{second}$; moderate: $279 \pm 114.4 \mathrm{~cm} /$ second; severe: $580.1 \pm 216.4 \mathrm{~cm} /$ second; intergroup $p<0.0001$ ). The results even after correction for significant confounding factors did not show any considerable change in the increasing trend of arterial stiffness.

Conclusion: This study establishes the functional deterioration of arteries in proportion to the severity of COVID-19.

Keywords: Arterial stiffness, COVID-19, COVID-19 ARDS, Endothelial injury, Intensive care unit.

Indian Journal of Critical Care Medicine (2021): 10.5005/jp-journals-10071-24000
\end{abstract}

\section{INTRODUCTION}

World Health Organization (WHO) declared coronavirus disease 2019 (COVID-19) as global pandemic and it is caused by severe acute respiratory syndrome coronavirus 2 (SARS-CoV-2). As of March 2021, in the second week, confirmed cases of COVID-19 in India alone are around 11.4 million, which include more than 159,000 deaths. This pandemic is affecting millions of people around the globe and cases are rising every day, leading to a public health emergency situation. Still the treatment options to manage this disease condition are hard to find by our medical professionals. Drugs like remdesivir or convalescent plasma have been tried under emergency use authorization without much success. Vaccines for COVID-19 have been under development as early as May 2020, ${ }^{1}$ and a few have been discovered. As of now, few vaccines are already in use. But the challenge of managing COVID-19 complications remains as new strains emerge and cause recent spikes in infections worldwide. The priority is to establish and understand the pathophysiology and relationship of arterial damage in COVID-19 patients. This enables the caregiver to guide the therapy and control long- and short-term complications in the cardiovascular system.

Some evidence may radically change the therapeutic strategy or give rise to a new therapy to improve the prognosis and lessen the burden on the healthcare facilities to a large extent. SARSCoV-2 infection affects the cardiovascular system at many levels. There is evident endothelial damage and inflammation due to COVID-19. ${ }^{2}$ There is reported evidence of cardiac infection ${ }^{3}$,
1,5Department of Trauma and Emergency, All India Institute of Medical Sciences, Patna, Bihar, India

${ }^{2}$ Department of CTVS, All India Institute of Medical Sciences, Patna, Bihar, India

3,6 Department of Anaesthesiology, All India Institute of Medical Sciences, Patna, Bihar, India

${ }^{4}$ Department of General Medicine, All India Institute of Medical Sciences, Patna, Bihar, India

${ }^{7}$ Department of Burns and Plastic Surgery, All India Institute of Medical Sciences, Patna, Bihar, India

${ }^{8}$ Director, All India Institute of Medical Sciences, Patna, Bihar, India

Corresponding Author: Neeraj Kumar, Department of Trauma and Emergency, All India Institute of Medical Sciences, Patna, Bihar, India, Phone: +91 08210104972, e-mail: neeraj.jnmc@gmail.com

How to cite this article: Kumar N, Kumar S, Kumar A, Bhushan D, Kumar A, Kumar A, et al. The COSEVAST Study Outcome: Evidence of COVID-19 Severity Proportionate to Surge in Arterial Stiffness. Indian J Crit Care Med 2021;25(10):1113-1119.

Source of support: Nil

Conflict of interest: None

endothelial injury, and endotheliitis over the multiple vascular beds ${ }^{4}$ in various patients after autopsy due to COVID-19. The European Society of Cardiology (ESC) establishes a two-way close association between COVID-19 and cardiovascular 
diseases (CVD). A preexisting cardiovascular system has a worse prognosis. ${ }^{5}$ Similar to primary inflammatory diseases, there are reports of immune-mediated vascular damage due to COVID-19 associated inflammation. ${ }^{6}$ There is an increased risk of cardiovascular events due to the overactivation of innate immunity following severe COVID-19 infection, presence of cytokine-release syndrome, and altered immune response and may result in indirect, immune-mediated damage to the systemic vasculature. ${ }^{7}$ Cardiovascular involvement is dominant in COVID-19 patients. The independent predictors of cardiovascular risk are arterial stiffness and enhanced pulse wave velocity (PWV). ${ }^{8-10}$ Due to stiffness of larger arteries, there may be mechanical fatiguability of the arterial wall leading to increased left ventricular afterload and altered coronary perfusion. ${ }^{11}$ However, the findings so far are not empirical that there is a compromise in the function of the arterial walls. A study to determine systemic inflammation and arterial stiffness in COVID-19 patients was designed. The study is justified by a comprehensive review article. $^{12}$

\section{Hypothesis and Objectives}

We hypothesized that mild, moderate, and severe categories of COVID-19 patients were associated with a corresponding increase in arterial stiffness. The main objective of the COVID-19 severity with arterial stiffness (COSEVAST) study was to evaluate the presence of arterial stiffness after COVID-19 infection. The primary endpoint was decided as estimated carotid-femoral PWV (cfPWV), an established biomarker of arterial stiffness.

Secondary objectives were as follows:

- To evaluate if the overall cardiovascular health is affected by measuring other markers, like age-normalized increase in aortic augmentation pressure (ANI_AugP), heart rate-normalized augmentation index ( $\mathrm{HRN}_{-}$Alx), age-normalized in cfPWV (ANI_cfPWV), in all three categories of mild, moderate, and severe COVID-19 patients.

- The objective is to study if arterial stiffness basal measures in COVID-19 patients at detection of infection can lead to assess the magnitude of potential confounding.

\section{Materials and Methods}

\section{Study Design}

The COSEVAST study is a nonrandomized observational study conducted at the All India Institute of Medical Sciences Patna, Bihar, India for COVID-19 patients admitted to intensive care units and medical wards. The entire duration of this study was 3 months, i.e., from November 2019 to January 2020.

\section{Ethical Considerations}

The COSEVAST study was a research study involving human participants with minimal risks and burdens. This study got approval from the Clinical Trials Registry of India having CTRI/2020/10/028489. We obtained Institutional Ethics Committee approval for conducting this study vide IEC letter No. AlIMS/Pat/ IEC/2020/595.

\section{Study Population}

All participants were confirmed COVID-19 with an RT-PCR test. This study included three categories of COVID-19 patients. The inclusion criteria were as follows:

- Age more than 18 years.
- Patients with a confirmed infection by SARS-CoV-2, requiring hospitalization in hospital wards and intensive care units.

The selected mild, moderate, and severe categories of patients were based on the latest National Institute of Health $(\mathrm{NIH})$ guidelines ${ }^{13}$ as per the following:

- Mild category: Patients with signs of vomiting, sore throat, nausea, headache, cough, fever, malaise, muscle pain, loss of taste or smell or both, and diarrhea, but with no evidence of dyspnea, shortness of breath, or abnormal chest imaging.

- Moderate category: Patients who have a saturation of oxygen $\left(\mathrm{SpO}_{2}\right) \geq 94 \%$ on room air, and on clinical and radiological imaging suggests the evidence of lower respiratory disease

- Severe category: Patients who have $\mathrm{PaO}_{2} / \mathrm{FiO}_{2}<300 \mathrm{~mm} \mathrm{Hg}$, or lung infiltrates $>50 \%$, saturation of oxygen $\left(\mathrm{SpO}_{2}\right)<94 \%$ on room air, and a respiratory rate of $>30$ breaths/minute.

The exclusion criteria were inability to provide written consent, pregnancy or breastfeeding, and known history of any of these diseases: diabetes mellitus, hypertension, coronary artery disease (CAD), stroke, neuropathy, peripheral arterial disease, kidney diseases, and any preexisting cardiovascular disorder.

\section{Methods}

\section{Measures of Arterial Stiffness}

By applying Framingham risk equations, the cardiovascular risk, atherosclerotic event, and number of cardiovascular risk factors (treatable vs nontreatable) were assessed based on arterial stiffness and PWV. ${ }^{14}$ The carotid intima-media thickness is the marker of atherosclerotic burden in the coronary arteries, and it is directly correlated with PWV. ${ }^{15}$ Arterial stiffness in this study is primarily measured in terms of PWV. Aortic and brachial PWV ${ }^{16}$ and arterial pressure augmentation index $(\mathrm{Alx})^{17}$ are independently related to the levels of inflammation, suggesting that inflammation plays a role in the regulation of arterial stiffening.

\section{Arterial Stiffness Assessment in Our Study}

There are various techniques to measure PWV; since COVID-19 is a contagious disease, minimal contact between patient and technician must be prioritized while conducting the test. It would be challenging for the observer to conduct the entire test while wearing a complete PPE kit. So, a test device, which lacks probe holding, avoids close contact and exposure time, while testing was needed for the study especially in these COVID-19 conditions. These requirements were fulfilled by using the medical device Periscope ${ }^{\mathrm{TM}}$ manufactured by Genesis Medical Systems, India. It was a noninvasive medical device used to measure brachial-ankle PWV (baPWV). It calculates the cfPWV, nearly equal to aortic PWV. ${ }^{18}$ The operator's manual of Periscope was used for performing the test. Firstly, over the ventral surface of both wrists and medial side of ankles, the electrocardiogram (ECG) electrodes were applied, and over both the upper and lower limbs, blood pressure (BP) cuffs were wrapped on brachial artery and tibial artery, respectively. All data were automatically stored in the computer for analysis of the ECG and pressure waveforms using integrated software algorithms. The Periscope device detects two lead ECGs (Lead I and II) along with pressure waveforms from four-limb locations, i.e., both brachial and ankles. The time difference between lowest initiation points of pulse pressure matching with QRS complex for calculating the pulse transit time (PTT). The software estimated cfPWV from the composite 
baPWV (Avg. baPWV) found out by averaging left and right baPWV as per the following validated equation: CfPWV $=0.8333^{*}$ (Avg. baPWV) -233.33

The established markers for arterial stiffness are aortic pressure values. Due to an increase in arterial stiffness, at the aortic root level, Periscope estimates the aortic pressures and the systolic pressure augmentation (AugP). The pressure augmentation index (Alx) values derived with Periscope device were very accurate and highly comparable with other internationally accepted noninvasive devices. ${ }^{20}$

\section{Sample Size}

Since COVID-19 is a completely new condition, sample size calculation cannot be based on preliminary data. In a study by Hofmann et al., ${ }^{21}$ the mean cfPWV values in CAD patients and healthy patients were $9.3 \pm 1.9 \mathrm{~m} / \mathrm{second}$ and $7.7 \pm 1.1 \mathrm{~m} / \mathrm{second}$, respectively. Thus, we estimated that the minimal detectable difference in PWV of $20 \%$ in different groups of patients with COVID-19; the sample size was calculated to be 16 in each group, taking an alpha error of 0.05 , power of $80 \%$, and $p$-value $\leq 0.05$ as statistically significant. Assuming a drop out of $5 \%$, we included 23 patients in each group (mild, moderate, and severe category).

\section{Statistical Analysis}

The Kolmogorov-Smirnov (KS) test was used for calculating the normal distribution of demographic data of the patient. By applying the nonparametric unpaired Student's " $t$ " test, we measure the difference of arterial stiffness among all three study groups. The normality and variance were not tested for the arterial stiffness measurements to determine whether the applied parametric tests were appropriate or not. Probability values of $p$ $<0.05$ were considered to indicate statistical significance. All $p$ values are two-tailed and expressed as mean value \pm standard deviation (SD). Statistical analysis was performed using the GraphPad PRISM software version 5.03 (GraphPad Software Inc., San Diego, California, USA).

\section{Results}

Recruited patients in the study were a total of 64 (22 female/42 male). Patient demographics are shown in Table 1. It was observed that all the patients were under healthy and normal body mass index (BMI) range. Lipid profile or blood sugar levels were not taken into consideration since the patients did not have any known comorbidity, age from 18 years and the maximum was 69 years considered. Though there was a difference between the mean values of age, height, and weight, it was not consistent and highly significant between the groups. All the demographic data passed the normality KS test except for gender (Table 1). For doing accurate comparison, we regularly measure the $\mathrm{PaO}_{2} \%$ and $\mathrm{SpO}_{2} \%$ for every patient while conducting the arterial stiffness test. The best way to quantify the COVID severity was to take the ratio of $\mathrm{SpO}_{2} \%$ and $\mathrm{FiO}_{2} \%$ as an indication of respiratory distress since removal of external oxygen support was not advisable in moderate and severe cases. The noninvasive measurement of $\mathrm{SpO}_{2} \%$ to $\mathrm{FiO}_{2} \%$ ratio closely relates with $\mathrm{PaO}_{2} \%$ to $\mathrm{FiO}_{2} \%$ ratio as per various studies and guidelines, ${ }^{22-24}$ especially in COVID-19 patients. The statistical analysis of the segregation criteria is shown in Table 2.

Arterial stiffness was analyzed in terms of the values of estimated cfPWV as a primary marker. It is well established that the value (functional) of arterial stiffness is a function of a person's current physiologic/chronologic age. ${ }^{25}$ Hence, an increase in cfPWV from the age-dependent normal value indicates the real increase in arterial stiffness from the normal level. A central AugP increase from age-dependent normal value and Alx are independent markers of arterial stiffness. All these measurements were analyzed for finding any relation between COVID-19 severity and arterial stiffness. The analysis of arterial stiffness parameters is given in Table 3, and Figure 1 shows (A) cfPWV in all three patient categories; (B) ANI_cfPWV; (C) ANI_AugP; and (D) HRN_Alx values. These measurements may be dependent on other confounding factors, like weight, height, gender, mean arterial pressure (MAP), and heart rate of the patient. To find out the effect of these factors on the results, we did an independent regression analysis of the

Table 1: Patient demographic characteristics as per COVID-19 severity

\begin{tabular}{|c|c|c|c|c|c|c|c|}
\hline \multirow[b]{2}{*}{ Parameter } & \multirow[b]{2}{*}{ Mild (1) } & \multirow[b]{2}{*}{ Moderate (2) } & \multirow[b]{2}{*}{ Severe (3) } & \multirow[b]{2}{*}{ Total } & \multicolumn{3}{|c|}{$p$ value } \\
\hline & & & & & Group 1-2 & Group 2-3 & Group 1-3 \\
\hline Sex (Male/Female) & $13 / 10$ & $18 / 3$ & $11 / 9$ & $42 / 22$ & - & - & - \\
\hline Age (years) & $37.87 \pm 13.36$ & $45.29 \pm 13.07$ & $50.50 \pm 12.56$ & $44.25 \pm 13.85$ & 0.0701 & 0.2007 & $0.0028^{*}$ \\
\hline Weight (Kg) & $61.96 \pm 13.32$ & $70.14 \pm 12.75$ & $59.05 \pm 10.12$ & $63.73 \pm 12.89$ & $0.0438^{*}$ & $0.0038^{*}$ & 0.4307 \\
\hline Height (cm) & $162.8 \pm 8.574$ & $167.9 \pm 8.402$ & $161.7 \pm 7.680$ & $164.1 \pm 8.555$ & 0.0541 & $0.0174^{*}$ & 0.6403 \\
\hline BMI $\left(\mathrm{Kg} / \mathrm{m}^{2}\right)$ & $23.18 \pm 3.547$ & $24.78 \pm 3.492$ & $22.47 \pm 2.532$ & $23.48 \pm 3.334$ & 0.1405 & $0.0207^{*}$ & 0.4605 \\
\hline
\end{tabular}

Values are expressed as mean \pm SD. ${ }^{*}$ Significant difference in mean values

Table 2: Patient categorization criteria as per COVID-19 severity

\begin{tabular}{lcccccc}
\hline & & & \multicolumn{3}{c}{ pvalue } \\
\cline { 5 - 7 } Parameter & Mild (1) & Moderate (2) & Severe (3) & Group 1-2 & Group 2-3 & Group 1-3 \\
\hline Respiration rate & $20.87 \pm 1.180$ & $22.33 \pm 4.139$ & $29.55 \pm 13.19$ & 0.1113 & $0.0219^{*}$ & $0.0031^{*}$ \\
$\mathrm{FiO}_{2}(\%)$ & $21.00 \pm 0.0$ & $35.48 \pm 12.68$ & $57.85 \pm 23.13$ & $<0.0001^{*}$ & $0.0004^{*}$ & $<0.0001^{* \$}$ \\
$\mathrm{SpO}_{2}(\%)$ & $97.30 \pm 1.428$ & $96.48 \pm 2.136$ & $96.50 \pm 2.236$ & 0.1350 & 0.7994 & 0.0861 \\
$\mathrm{SPO}_{2} / \mathrm{FiO}_{2}$ ratio & $463.4 \pm 6.801$ & $304.1 \pm 101.6$ & $195.9 \pm 82.33$ & $<0.0001^{*}$ & $0.0006^{*}$ & $<0.0001^{*}$ \\
\hline
\end{tabular}

Values are expressed as mean \pm SD. "Significant difference in mean values. ${ }^{\$}$ Mann-Whitney " $t$ " test 

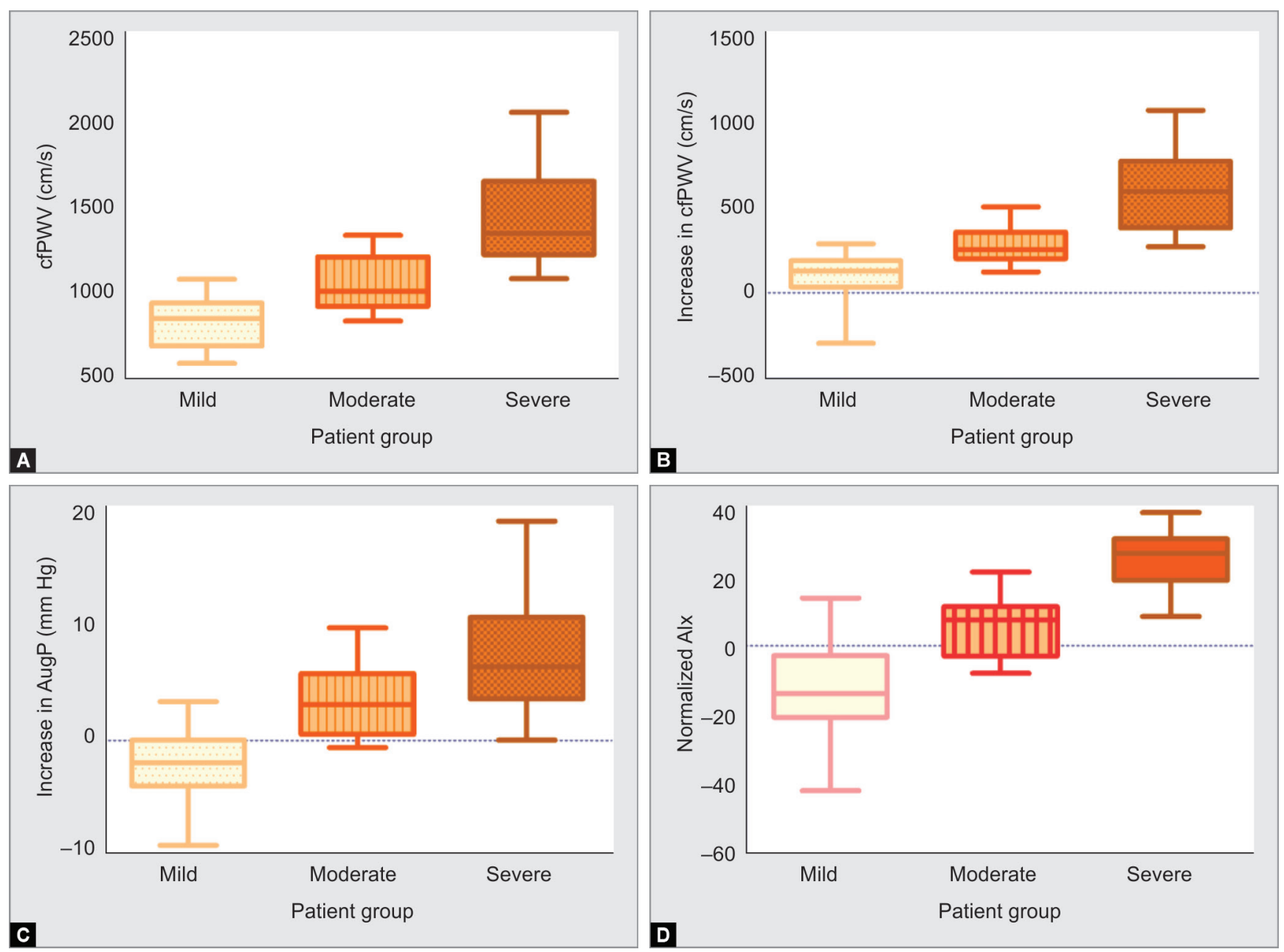

Figs $1 \mathrm{~A}$ to $\mathrm{D}$ : It shows cfPWV in all three patient categories, age-normalized increase in cfPWV, age-normalized increase in aortic augmentation pressure, and HR-normalized Alx values

Table 3: Patient arterial stiffness measures as per COVID-19 severity

\begin{tabular}{lrrrrrr}
\hline & & & & \multicolumn{3}{c}{$p$ value } \\
\cline { 5 - 7 } Parameter & \multicolumn{1}{c}{ Mild (1) } & Moderate (2) & Severe (3) & Group 1-2 & Group 2-3 & Group 1-3 \\
\hline cfPWV (cm/second) & $829.1 \pm 139.2$ & $1067 \pm 152.5$ & $1416 \pm 253.9$ & $<0.0001^{*}$ & $<0.0001^{*}$ & $<0.0001^{*}$ \\
cfPWV increase $^{*}(\mathrm{~cm} /$ second) & $101.2 \pm 126.1$ & $279 \pm 114.4$ & $580.1 \pm 216.4$ & $<0.0001^{*}$ & $<0.0001^{*}$ & $<0.0001^{*}$ \\
AugP increase $^{*}(\mathrm{~mm} \mathrm{Hg})$ & $-1.891 \pm 2.817$ & $3.212 \pm 3.124$ & $7.246 \pm 4.908$ & $<0.0001^{*}$ & $0.0031^{*}$ & $<0.0001^{*}$ \\
Alx & $4.670 \pm 13.07$ & $22.16 \pm 7.833$ & $34.24 \pm 8.467$ & $<0.0001^{*}$ & $<0.0001^{*}$ & $<0.0001^{*}$ \\
\hline
\end{tabular}

Values are expressed as mean \pm SD. ${ }^{*}$ Significant difference in mean values. ${ }^{\#}$ Age normalized

confounding factors with cfPWV. Table 4 shows the significance of possible confounding factors in cfPWV.

This regression analysis found that weight, MAP, and age make a significant contribution to cfPWV apart from the severity grade in our dataset. Since the normal value of arterial stiffness progressively increases with age, we corrected the observed values of cfPWV in our dataset for MAP and weight, and the other significant factors, as per the age-based predicted values, found a significant increasing trend of cfPWV among the three groups, viz. mild, moderate, and severe. We treated all other measurements for regression analysis and found that ANI_cfPWV is affected only by MAP after eliminating the insignificant factors. Similarly, AugP depends on MAP, HR, and age; Alx depends on MAP and age for the data in our study. After correction for the confounding variables, the values of these measurements showed a significant correlation between the patient severity and values of these parameters.

A study done earlier shows that both Alx values and aortic root systolic pressure (AoSysP) are dependent on heart rate. ${ }^{26}$ To rule out the role of heart rate in our findings, Alx values were normalized for HR. After correction for the confounding factors, there were changes in the values of the measurements. However, the corrected values showed increasing trends similar to uncorrected values. The significance of the differences in the mean values was also more or less unaffected after the correction. Table 5 shows the statistical analysis of the measurements, corrected for significant confounding factors, and Figure 2 shows the results of arterial stiffness measurements of COVID-19 patients after correction in graphical format. 

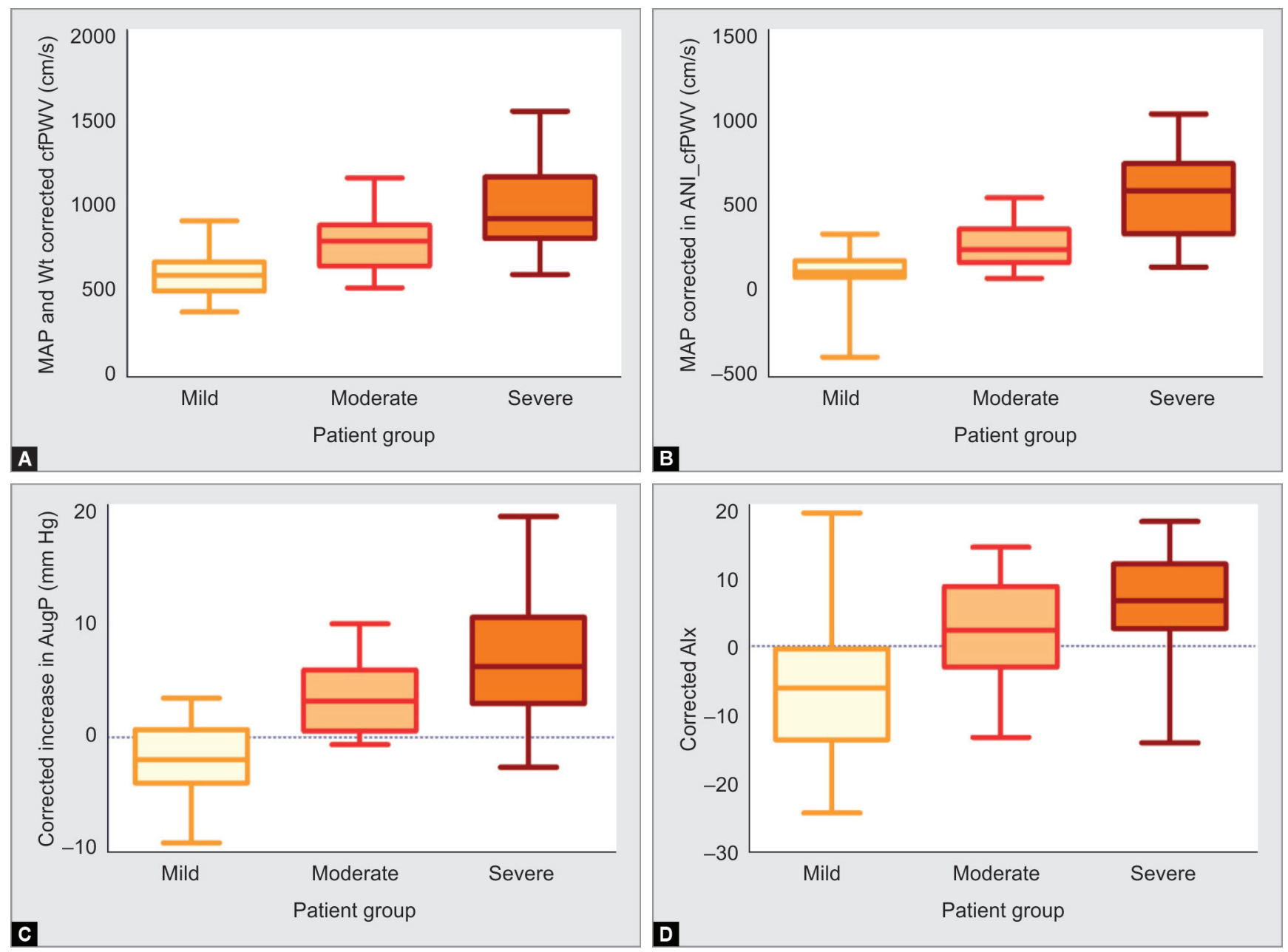

Figs 2A to D: It shows the results of arterial stiffness measurements of COVID-19 patients after correction in graphical format

Table 4: Significance of possible confounding factors in cfPWV

\begin{tabular}{llcc}
\hline Possible confounding variables & tratio & p value & Level of significance \\
\hline Age (years) & 6.644 & $<0.0001$ & Yes \\
Gender & 0.3518 & 0.7263 & No \\
Weight $(\mathrm{kg})$ & 2.441 & 0.0178 & Yes \\
Height $(\mathrm{cm})$ & 1.133 & 0.2621 & No \\
Brachial average MAP (mm Hg) & 4.5 & $<0.0001$ & Yes \\
Heart rate (bpm) & 0.04871 & 0.9613 & No \\
\hline
\end{tabular}

Only age, weight, and brachial average MAP make significant contribution in cfPWV

Table 5: Patient arterial stiffness measures after correction for significant or known confounding factors as per COVID-19 severity

\begin{tabular}{|c|c|c|c|c|c|c|}
\hline \multirow[b]{2}{*}{ Parameter } & \multirow[b]{2}{*}{ Mild (1) } & \multirow[b]{2}{*}{ Moderate (2) } & \multirow[b]{2}{*}{ Severe (3) } & \multicolumn{3}{|c|}{$p$ value } \\
\hline & & & & Group 1-2 & Group 2-3 & Group 1-3 \\
\hline cfPWV (cm/second) & $606.4 \pm 125.9$ & $781.5 \pm 165.1$ & $982.9 \pm 243.1$ & $0.0003^{*}$ & $0.0034^{*}$ & $<0.0001^{*}$ \\
\hline cfPWV increase ${ }^{\#}$ (cm/second) & $118.2 \pm 142.2$ & $273.2 \pm 128.8$ & $558.3 \pm 241.0$ & $0.0005^{*}$ & $<0.0001^{*}$ & $<0.0001^{*}$ \\
\hline AugP increase ${ }^{\#}(\mathrm{~mm} \mathrm{Hg})$ & $-2.230 \pm 3.237$ & $0.3146 \pm 2.968$ & $2.8 \pm 4.142$ & $0.0097^{*}$ & $0.0325^{*}$ & $<0.0001^{*}$ \\
\hline Alx & $-6.573 \pm 10.06$ & $2.038 \pm 7.222$ & $6.069 \pm 7.979$ & $<0.0024^{*}$ & $0.0976^{*}$ & $<0.0001^{*}$ \\
\hline HR-normalized Alx & $-13.34 \pm 14.18$ & $5.656 \pm 8.610$ & $24.80 \pm 7.745$ & $<0.0001^{*}$ & $<0.0001^{*}$ & $<0.0001^{*}$ \\
\hline
\end{tabular}

Values are expressed as mean \pm SD. ${ }^{*}$ Significant difference in mean values. ${ }^{\#}$ Age normalized 


\section{Discussion}

The SARS-CoV-2 binds its viral spike (S) protein to angiotensinconverting enzyme 2 (ACE2) on the host cell's surface to gain entry into the cell. The full endothelial layer is affected throughout the body and the disease called endotheliitis sets in due to COVID-19 (SARS-CoV-2) infection. The Lancet article ${ }^{27}$ shows clear images with endothelial layer involvement. Chen et al. ${ }^{28}$ in their epidemiological studies show that patients with COVID-19 admitted to hospital/ intensive care unit frequently present with accompanying conditions, such as advanced age, diabetes, hypertension, and cardiovascular diseases, suggesting compromised endothelial integrity may possibly be playing a major part in (SARS-CoV-2) COVID-19 mortality.

Theestimated cfPWV, which we observed in severe(SARS-CoV-2) COVID-19 patients, was higher than the CfPWV in patients with long-term chronic diseases like diabetes mellitus, CAD, and end-stage renal disease. For example, in CAD patients, it was $1204 \pm 301.8 \mathrm{~cm} /$ second. ${ }^{19,20}$ This indicates that (SARS-CoV-2) COVID-19 damage to the patient vasculature within less than 15 days is comparable to the damage caused by CAD in the number of years. Conducted COSEVAST study had strict exclusion criteria. Patients with known hypertension and diabetes were not included. Patients ailing with existing cardiovascular diseases and related surgical intervention/therapy were excluded. Patients with a history of smoking and increased obesity were also excluded. This eliminated the possible basal deviation of arterial stiffness from the normal vasculature. The validity of our study findings was more accurate because we strictly followed the exclusions criteria to avoid selection bias. An ongoing similar large-scale, multicentric longitudinal study on more than 2,500 people (COVID-19 ARTErial Stiffness and vascular AgiNg (ARTESIAN study) ${ }^{29}$ identified the association between early vascular aging and its relationship with COVID- 19 based on its disease severity. The COSEVAST study will help to determine efficacious therapy in reducing (SARS-CoV-2) COVID-19 disease complications. Based on our scientific literature search, this is the first study which observed that arterial inflammation in COVID-19 patients in terms of noninvasive measurement of arterial stiffness, like PWV, has brought significant functional changes in the vascular beds.

\section{LIMITATIONS}

Since it was an observational study only, the patients were not abstained from the standard therapy to reduce the (SARS-CoV-2) COVID-19 symptoms. Medications were uniform intragroup and did not include any drug that affects arterial stiffness. Especially, the therapy of moderate and severe groups was identical except for the difference in oxygen supplementation volume. The sample size of patients was small given the limitations on entry in a COVID ward for any test procedure.

\section{Conclusion}

It can be reasonably stated that this study suggests that a noninvasive measure of arterial stiffness may be useful as an independent severity marker in COVID-19 patients. There is a strong indication that the management of arterial stiffness by titration of therapeutics may possibly reduce acute cardiovascular complications and mortality in (SARS-CoV-2) COVID-19 patients.

\section{ACKNOWLedgments}

This scientific abstract was presented in CRITICARE 2021 (Virtual Conference) organized by the Indian Society of Critical Care Medicine dated February 26 to 28, 2021, and this abstract bags the best oral paper award at this conference with Conference Abstract ID: 48.

There are many people involved in our research project who are not authors but have provided valuable contributions namely our entire residents, technical staffs, and nursing officers for their expertise and assistance throughout all aspects of our study, especially during the COVID-19 pandemic

\section{OrCID}

Neeraj Kumar (1) https://orcid.org/0000-0002-9161-7000

Sanjeev Kumar (1) https://orcid.org/0000-0003-4867-9326

Abhyuday Kumar (1) https://orcid.org/0000-0002-9247-6713

Divendu Bhushan (1) https://orcid.org/0000-0002-8718-5890

Amarjeet Kumar $\odot$ https://orcid.org/0000-0002-4272-5750

Ajeet Kumar $\odot$ https://orcid.org/0000-0002-1464-6684

Veena Singh $\odot$ https://orcid.org/0000-0002-6300-7811

Prabhat K Singh (1) https://orcid.org/0000-0003-1888-4679

\section{List of Abbreviations}

AS-Arterial Stiffness

RT-PCR-Reverse Transcription Polymerase Chain Reaction

cf-Carotid Femoral

CVD-Cardiovascular Disease

HR-Heart rate

PP_-Pulse Pressure

PWV-Pulse Wave Velocity

SBP-Systolic Blood Pressure

SE-Standard Error

$\mathrm{NIH}$ - National Institute of Health

COVID 19-Coronavirus disease 2019

SARS-Severe Acute Respiratory Syndrome

\section{References}

1. Sarvepalli D. Coronavirus disease 2019: a comprehensive review of etiology, pathogenesis, diagnosis, and ongoing clinical trials. Cureus 2020;12(5):e8076. DOI: 10.7759/cureus.8076.

2. Varga Z, Flammer AJ, Steiger $P$, Haberecker $M$, Andermatt $R$, Zinkernagel AS, et al. Endothelial cell infection and endotheliitis in COVID-19. Lancet 2020;395(10234):1417-1418. DOI: 10.1016/S01406736(20)30937-5.

3. Lindner D, Fitzek A, Brauninger H, Aleshcheva G, Edler C, Meissner K, et al. Association of cardiac infection with SARS-CoV-2 in confirmed COVID-19 autopsy cases. JAMA Cardiol 2020;5(11):1281-1285. DOI: 10.1001/jamacardio.2020.3551.

4. Ding $Y$, Wang $H$, Shen $H$, Li Z, Geng J, Han H, et al. The clinical pathology of the severe acute respiratory syndrome (SARS): a report from China. J Pathol 2003;200(3):282-289. DOI: 10.1002/path.1440.

5. Guzik TJ, Mohiddin SA, Dimarco A, Patel V, Savvatis K, Marelli-Berg FM, et al. COVID-19 and the cardiovascular system implications for risk assessment, diagnosis, and treatment options Cardiovasc Res 2020;116(10):1666-1687. DOI: 10.1093/cvr/cvaa106.

6. Zanoli L, Briet M, Empana JP, Cunha PG, Mäki-Petäjä KM, Protogerou $A D$, et al. Vascular consequences of inflammation: a position statement from the ESH Working Group on Vascular Structure and Function and the ARTERY Society. J Hypertens 2020;38(9):1682-1698. DOI: $10.1097 / \mathrm{HJH} .0000000000002508$. 
7. He L, Ding $Y$, Zhang $Q$, Che $X, H e ~ Y$, Shen $H$, et al. Expression of elevated levels of pro-inflammatory cytokines in SARS-CoV-infected ACE2+ cells in SARS patients: relation to the acute lung injury and pathogenesis of SARS. J Pathol 2006;210(3):288-297. DOI: 10.1002/ path.2067.

8. Stefanadis C, Dernellis J, Tsiamis E, Stratos C, Diamantopoulos L, Michaelides $A$, et al. Aortic stiffness is a risk factor for recurrent acute coronary events in patients with ischaemic heart disease. Eur Heart J 2000;21(5):390-396. DOI: 10.1053/euhj.1999.1756.

9. Stefanadis C, Wooley CF, Bush CA, Kolibash AJ, Boudoulas H. Aortic distensibility abnormalities in coronary artery disease. Am J Cardiol 1987;59(15):1300-1304. DOI: 10.1016/0002-9149(87)90908-8.

10. Blacher J, Asmar R, Djane S, London GM, Safar ME. Aortic pulse velocity as a marker of cardiovascular risk in hypertensive patients. Hypertension 1999;33(5):1111-1117. DOI: 10.1161/01.hyp.33.5.1111.

11. Nichols WW, O'Rourke MF, editors. McDonald's Blood Flow in Arteries. 4th ed. London, UK: Edward Arnold; 1998. p. 170-222, 284-315, 347-395, 450-476.

12. Sparks MA, South AM, Badley AD, Baker-Smith CM, Batlle D, Bozkurt B, et al. Severe acute respiratory syndrome coronavirus 2, COVID-19, and the Renin-Angiotensin system. Hypertension 2020;76(5):1350-1367. DOI: 10.1161/HYPERTENSIONAHA.120.15948.

13. Clinical Presentation of People with SARS-CoV-2 Infection Last Updated: October 9, 2020. Available from: https://www. covid19treatmentguidelines.nih.gov/overview/clinical-presentation.

14. Vogel RA, Benitez RM. Non-invasive assessment of cardiovascular risk: from Framingham to the future. Rev Cardiovasc Med 2000;1(1):34-42. PMID: 12457150.

15. Wofford JL, Kahl FR, Howard GR, McKinney WM, Toole JF, et al. 3rd. Relation of extent of extracranial carotid artery atherosclerosis as measured by B-mode ultrasound to the extent of coronary atherosclerosis. Arterioscler Thromb 1991;11(6):1786-1794. DOI: 10.1161/01.atv.11.6.1786.

16. Kampus P, Kals J, Ristimae T, Muda P, Ulst K, Zilmer K, et al. Augmentation index and carotid intima-media thickness are differently related to age, C-reactive protein and oxidized lowdensity lipoprotein. J Hypertens 2007;25(4):819-825. DOI: 10.1097/ HJH.0b013e328014952b.

17. Yasmin, McEniery CM, Wallace S, Mackenzie IS, Cockcroft JR, Wilkinson IB. C-reactive protein is associated with arterial stiffness in apparently healthy individuals. Arterioscler Thromb Vasc Biol 2004;24(5): 969-974. DOI: 10.1161/01.ATV.zhq0504.0173.

18. Naidu MU, Reddy BM, Yashmaina S, Patnaik AN, Rani PU. Validity and reproducibility of arterial pulse wave velocity measurement using new device with oscillometric technique: a pilot study. Biomed Eng Online 2005;4:49. DOI: 10.1186/1475-925X-4-49.
19. Shridhar Y, Naidu MU, Usharani P, Raju YS. Non-invasive evaluation of arterial stiffness in patients with increased risk of cardiovascular morbidity: a cross sectional study. Indian J Pharmacol 2007;39(6): 294-298. DOI: 10.4103/0253-7613.39150.

20. Naidu MUR, Prabhakar RC. Non-invasive measurement of aortic pressure in patients: comparing pulse wave analysis and applanation tonometry. Indian J Pharmacol 2012;44(2):230-233. DOI: 10.4103/0253-7613.93855.

21. Hofmann B, Riemer M, Erbs C, Plehn A, Navarrete Santos A, Wienke $A$, et al. Carotid to femoral pulse wave velocity reflects the extent of coronary artery disease. J Clin Hypertens (Greenwich) 2014;16(9):629-633. DOI: 10.1111/jch.12382.

22. Lu X, Jiang L, Chen T, Wang Y, Zhang B, Hong Y, et al. Continuously available ratio of $\mathrm{SpO}_{2} / \mathrm{FiO}_{2}$ serves as a non-invasive prognostic marker for intensive care patients with COVID-19. Respir Res 2020;21(1):194. DOI: 10.1186/s12931-020-01455-4.

23. Bilan N, Dastranji A, Ghalehgolab Behbahani A. Comparison of the $\mathrm{SpO}_{2} / \mathrm{FiO}_{2}$ ratio and the $\mathrm{PaO}_{2} / \mathrm{FiO}_{2}$ ratio in patients with acute lung injury or acute respiratory distress syndrome. J Cardiovasc Thorac Res 2015;7(1):28-31. DOI: 10.15171/jcvtr.2014.06.

24. American Heart Association-CPR and First Aid Emergency Cardiovascular Care. Oxygenation and ventilation of COVID-19 patients module 4: ventilation management. Available from: https:// cpr.heart.org/en/resources/coronavirus-covid19-resources-for-cprtraining/oxygenation-and-ventilation-of-covid-19-patients.

25. Determinants of pulse wave velocity in healthy people and in the presence of cardiovascular risk factors: "establishing normal and reference values". The Reference Values for Arterial Stiffness' Collaboration. Eur Heart J 2010;31(19):2338-2350. DOI: 10.1093/ eurheartj/ehq165.

26. Wilkinson IB, MacCallum H, Flint L, Cockcroft JR, Newby DE, Webb DJ. The influence of heart rate on augmentation index and central arterial pressure in humans. J Physiol 2000;525.1:263-270. DOI: 10.1111/j.1469-7793.2000.t01-1-00263.x.

27. Varga Z, Flammer AJ, Steiger P, Haberecker M, Andermatt $R$, Zinkernagel AS, et al. Endothelial cell infection and endotheliitis in COVID-19. Lancet 2020;395(10234):P1417-P1418. DOI: 10.1016/S01406736(20)30937-5.

28. Chen T, Wu D, Chen H, Yan W, Yang D, Chen G, et al. Clinical characteristics of 113 deceased patients with coronavirus disease 2019: retrospective study. BMJ 2020;368:m1091. DOI: 10.1136/bmj.m1091.

29. Bruno1 RM, Spronck B, Hametner B, Hughes A, Lacolley P, Mayer CC. Covid-19 effects on ARTErial StIffness and Vascular AgeiNg: CARTESIAN study rationale and protocol. Artery Res 2021;27(2):59-68. DOI: 10.2991/artres.k.201124.001. Available from: https://www. atlantispress.com/journals/artres/125947864/view\#AFF1. 